\title{
SN 2001em: Evidence for a dense circumstellar environment
}

\author{
Frank Schinzel* \\ University of New Mexico, Albuquerque, USA \\ Now: Max-Planck-Institut für Radioastronomie, Germany \\ E-mail: schinzel@mpifr.de
}

\section{Gregory Taylor}

Department of Physics and Astronomy, University of New Mexico, USA

G.B. Taylor is also an Adjunct Astronomer at the National Radio Astronomy Observatory

E-mail: gbtayloreunm.edu

\section{Christopher Stockdale}

Marquette University, USA

E-mail: christopher.stockdale@marquette.edu

\section{Jonathan Granot}

Centre for Astrophysics Research, University of Hertfordshire, UK

E-mail: j.granotaherts.ac.uk

\section{Enrico Ramirez-Ruiz}

Department of Astronomy and Astrophysics, University of California, Santa Cruz, USA

E-mail: enrico@ucolick.org

SN 2001em is a peculiar supernova, originally classified as Type Ib/c. About two years after the occurrence of the $\mathrm{SN}$ it was detected in the radio, showing a rising radio flux with an optically thin spectral slope, and it also displayed a large X-ray luminosity $\left(\sim 10^{41} \mathrm{erg} \mathrm{s}^{-1}\right)$. About 3 years after its discovery the optical spectrum of SN $2001 \mathrm{em}$ showed a broad $\mathrm{H} \alpha$ line, and it was therefore reclassified as Type IIn. We constrained its proper motion and expansion velocity by analyzing four epochs of VLBI observations, extending out to 5.4 years after the SN. The SN is still unresolved 5.4 years after the explosion. For the proper motion we obtain $(23,000 \pm 30,000) \mathrm{km} \mathrm{s}^{-1}$ while our $2 \sigma$ upper limit on the expansion velocity is $6000 \mathrm{~km} \mathrm{~s}^{-1}$. These limits are somewhat tighter than those derived by Bietenholz \& Bartel. VLA observations of the radio flux density, at 8.46 $\mathrm{GHz}$, show a decay as $t^{-1.23 \pm 0.40}$ starting $\sim 2.7$ years after the SN. Collectively, the observations suggest interaction of the SN ejecta with a very dense circumstellar medium, though the implied opacity constraints still present a challenge.

The 9th European VLBI Network Symposium on The role of VLBI in the Golden Age for Radio Astronomy and EVN Users Meeting

September 23-26, 2008

Bologna, Italy

\footnotetext{
* Speaker.
} 


\section{Introduction}

On September 15, 2001, Papenkova \& Li [6] reported the discovery of an apparent Supernova (SN) in the galaxy UGC 11794 (RA: 21h42m22.9s DEC: 12d29m54s; $\mathrm{z}=0.019493 \mathrm{~J} 2000.0$ ). The measured redshift corresponds to a proper distance of $D_{p}=83.14 \mathrm{Mpc}$ and an angular distance of $D_{A}=81.55 \mathrm{Mpc}\left(\mathrm{H}_{0}=70 \mathrm{~km} \mathrm{~s}^{-1} \mathrm{Mpc}^{-1}\right.$, and $\Omega_{\Lambda}=1-\Omega_{M}=0.72$ [5]). It was detected in the radio about 2 years after the explosion [10]. It also showed a high X-ray luminosity of $\sim 10^{41} \mathrm{erg} \mathrm{s}^{-1}$ at $0.5-8 \mathrm{keV}$ [7]. This made it a good candidate for harboring a bipolar relativistic jet pointing away from our line of sight [4], which has by then decelerated to mildly relativistic velocities. This led to the classification of Type $\mathrm{Ib} / \mathrm{c}$. However, later observations, about 3 years after the explosion Soderberg et al. (2004) [9], showed broad H $\alpha$ (FWHM $40 \AA=1800 \mathrm{~km} \mathrm{~s}^{-1}$ ) lines that are not typical of Type Ib/c SN. An alternative explanation was suggested by [3], who developed a model in which regular Newtonian ejecta from the SN explosion collides with a dense, massive circumstellar shell (CS).

\section{Observations}

We observed SN 2001em with the HSA ${ }^{1}$. The observation was conducted on February 5th, 2007 with a total time of 10 hours at $8.4 \mathrm{GHz}$ (bandwidth $32 \mathrm{MHz}$, total recording bit rate 256 Mbit s$^{-1}$ ). VLBI data was correlated with the NRAO VLBA processor at Socorro. Analysis was done with AIPS $^{2}$ and Caltech's Difmap for modeling. In addition we also used the VLA (in A configuration) observation on February 18th 2007, which observed the continuum of SN 2001em in L, C and K-Band over a total time of about an hour. The three previous VLBA/HSA observations from July 2004 [11], November 2004 [1] and May 2006 [2] were re-reduced in a consistent fashion.

\section{Results}

Position and Proper Motion: We obtained the results for absolute position, thus for proper motion, by fitting the fully calibrated and phase-referenced $(u, v)$ plane data, of the observations from 4 epochs, to a circular Gaussian model. This is a good fit for a point source, as we did not see any resolved extended emission.

We obtained a proper motion of $(0.030 \pm 0.037)$ mas $\mathrm{year}^{-1}$ in R.A. and $(-0.053 \pm 0.071)$ mas year ${ }^{-1}$ in Dec., which gives a total proper motion of $v=(24,000 \pm 32,000) \mathrm{km} \mathrm{s}^{-1}$. This confirms the result obtained by Bietenholz \& Bartel (2007) [2] based on observations from two epochs separated by 1.5 years.

Expansion Limit of the Radio Emitting Region: Again we used the fully calibrated and phasereferenced $(u, v)$ plane data. The resolution of our observation was $(2.02 \times 0.858)$ mas at $-7.17^{\circ}$. A precise determination of the upper limit for the size of SN 2001em was obtained using MonteCarlo simulation ( $u, v$ plane fitting). Thus we obtain an upper limit diameter of the radio emitting region from Feb. 4th 2007 of 0.16 mas [8].

\footnotetext{
${ }^{1}$ High Sensitivity Array, consisting of the Very Long Baseline Array, phased Very Large Array, Arecibo Radio Telescope, Robert C. Byrd Green Bank Telescope, and Effelsberg Radio Telescope

${ }^{2}$ NRAO's Astronomical Image Processing System
} 
Assuming this linear expansion behavior since its discovery on 2001.704, we find a $2 \sigma$ upper limit for the expansion velocity of 0.015 mas year $^{-1}$, which corresponds to $\mathrm{v}_{\exp } \leq 6,000 \mathrm{~km} \mathrm{~s}^{-1}$.

Bietenholz \& Bartel (2007) [2] report a $2 \sigma$ upper limit for the average expansion velocity since the explosion of $25,800 \mathrm{~km} \mathrm{~s}^{-1}$. For that same epoch, when reanalyzing the same data we obtain a $2 \sigma$ upper limit of $8,100 \mathrm{~km} \mathrm{~s}^{-1}$.

Radio Lightcurve: Many multifrequency observations of SN 2001em have been conducted with the VLA since 2003. From the peak brightness the $8.46 \mathrm{GHz}$ peak luminosity is $(1.54 \pm 0.10) \times 10^{28}$ $\mathrm{erg} \mathrm{s}^{-1} \mathrm{~Hz}^{-1}$. We see an overall power law decay in the flux densities since its peak around 2004 (or $\sim 2.7 \mathrm{yr}$ since the $\mathrm{SN}$ ) with an index of $\alpha=1.23 \pm 0.40$, i.e. $F_{V} \propto t^{-\alpha}$. In addition the $8 \mathrm{GHz}$ lightcurve shows a slight re-brightening about one year after the peak brightness.

\section{Summary}

The radio properties of SN 2001em are consistent with a type II SN with a significant CSM, blastwave interaction. At the current rate of brightness decay and the expected increased recording rates of the VLBA/HSA we should be able to still detect it for the next 10-20 years and eventually resolve the slowly expanding shell of the SN. A detailed discussion of the methodology and implications of the results can be found in Schinzel et al. (2008) [8].

\section{References}

[1] M. F. Bietenholz and N. Bartel, An Upper Limit on the Expansion Velocity of Gamma-Ray Burst Candidate SN 2001em, Astrophys. J., Lett. 625 (2005), L99-L102.

[2] M. F. Bietenholz and N. Bartel, SN 2001em: No Jet-driven Gamma-Ray Burst Event, Astrophys. J., Lett. 665 (2007), L47-L50.

[3] N. N. Chugai and R. A. Chevalier, Late Emission from the Type Ib/c SN 2001em: Overtaking the Hydrogen Envelope, Astrophys. J. 641 (2006), 1051-1059.

[4] J. Granot and E. Ramirez-Ruiz, The Case for a Misaligned Relativistic Jet from SN 2001em, Astrophys. J., Lett. 609 (2004), L9-L12.

[5] G. Hinshaw, et al., Five-Year Wilkinson Microwave Anisotropy Probe (WMAP) Observations: Data Processing, Sky Maps, and Basic Results, ArXiv e-prints (2008).

[6] M. Papenkova, et al., Supernova 2001em in UGC 11794, IAU Circ. 7722 (2001), 1.

[7] D. Pooley and W. H. G. Lewin, Supernova 2001em in UGC 11794, IAU Circ. 8323 (2004), 2.

[8] Schinzel, F. K., et al. SN 2001em: NOT SO FAST, 2009, Astrophys. J., 691 (2009), 1380-1386

[9] A. M. Soderberg, A. Gal-Yam, and S. R. Kulkarni, Type Ic SN2001em (off-axis GRB jet?), optical spectrum., GRB Coordinates Network 2586 (2004), 1.

[10] C. J. Stockdale, et al., Supernova 2001em in UGC 11794, IAU Circ. 8282 (2004), 2.

[11] C. J. Stockdale, et al., VLBA Observations of SN 2001em: Supernova, Misdirected Gamma-Ray Burster, or Both?, Bulletin of the American Astronomical Society, Bulletin of the American Astronomical Society, vol. 36, December 2004, pp. 1464-+. 